\title{
Influence of experimental conditions on atom column visibility in energy dispersive X-ray spectroscopy
}

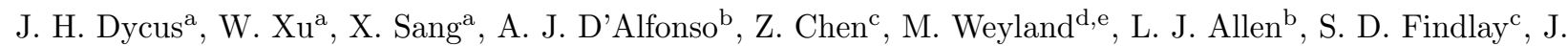 \\ M. LeBeau ${ }^{\mathrm{a}}$ \\ ${ }^{a}$ Department of Materials Science and Engineering, North Carolina State University, 911 Partners Way Engineering Building \\ 1, Raleigh $N C, 27606$ \\ ${ }^{b}$ School of Physics, University of Melbourne, Parkville, Victoria 3010, Australia \\ ${ }^{c}$ School of Physics and Astronomy, Monash University, Clayton, Victoria 3800, Australia \\ ${ }^{d}$ Monash Centre for Electron Microscopy, Monash University, Clayton, Victoria 3800, Australia \\ ${ }^{e}$ Department of Materials Science and Engineering, Monash University, Clayton, Victoria 3800, Australia
}

\begin{abstract}
Here we report the influence of key experimental parameters on atomically resolved energy dispersive X-ray spectroscopy (EDX). In particular, we examine the role of the probe forming convergence semi-angle, sample thickness, lattice spacing, and dwell/collection time. We show that an optimum specimen-dependent probe forming convergence angle exists to maximize the signal-to-noise ratio of the atomically resolved signal in EDX mapping. Furthermore, we highlight that it can be important to select an appropriate dwell time to efficiently process the X-ray signal. These practical considerations provide insight for experimental parameters in atomic resolution energy dispersive X-ray analysis.

Keywords: Scanning transmission electron microscopy (STEM); atomic resolution; energy-dispersive X-ray spectroscopy; electron channeling; probe convergence angle.
\end{abstract}

\section{Introduction}

Scanning transmission electron microscopy (STEM) has become a powerful tool for both structural and elemental quantification at the atomic scale. Energy dispersive X-ray spectroscopy (EDX) provides element specific maps with localized signal, providing directly interpretable elemental mapping [1,2]. Recent advances in X-ray detector technology have produced a significant increase in the collection solid angle, making atomic resolution EDX mapping routinely feasible $[3,4,5,6]$. Quantification of the atomically resolved signal has thus received considerable attention with the aim of determining composition on a column-by-column basis $[5,7,8,9]$. And while the effects of convergence angle have been studied on an absolute intensity scale without atomic resolution[10], the consequences of experimental parameters on the atomic resolution signal have yet to be reported. Many issues limit the quantification of EDX, including low signal generation and collection efficiency, fluorescence yields, X-ray absorption, detector geometry effects $[9,11,12]$, and the elastic and thermal scattering of the probe $[5,13]$. 
To approach quantitative atomic resolution EDX mapping and to understand other issues for quantification, overcoming poor overall signal must first be addressed. By increasing visibility for the atom column signal above the background, quantification of EDX maps can be performed with increased precision. It is important to note that the signal-to-noise ratio mentioned throughout the study refers to the statistical signal-to-noise level in a map rather than the peak-to-Bremsstrahlung background ratio in a spectrum. A method for improving the signal-to-noise ratio at atomic resolution is to use "lattice averaging" in EDX maps with each template encompassing a single or several unit cells $[7,14]$. This approach results in maps with significantly enhanced signal-to-noise ratios, providing a method towards greater quantitative precision [7]. Although the signal-to-noise is increased by averaging, the atomically resolved elemental signal is likewise averaged. Differences between individual atom column chemistry from the original map is then lost. Alternative solutions are thus required to optimize the X-ray signal and maximize the contrast of elemental signal above the noise.

In this article, we report the influence of experimental parameters on X-ray generation in atomic resolution EDX mapping. We consider the effects of the probe-forming convergence angle, specimen thickness, lattice spacing and dwell time on the total collected X-ray yield from the atom column (AC) signal and the signal between atom columns, as will henceforth be referred to as the non-atom column (non-AC) signal. Experimental results regarding intensities from AC and non-AC signals are compared with simulations for each condition.

\section{Materials and methods}

\subsection{Sample preparation and instrumentation}

Single crystal samples of $\langle 001\rangle \mathrm{SrTiO}_{3}(\mathrm{STO})$ were first thinned by mechanical polishing using an Allied Multiprep [15], then ion milled to electron transparency using a Fischione 1050 system stepped from $2 \mathrm{keV}$ to $200 \mathrm{eV}$. A probe-corrected, monochromated FEI G2 Titan 60-300 kV STEM/TEM operated at $200 \mathrm{kV}$ was used for imaging and spectroscopy. Probe-forming convergence semi-angles of either $13.5 \mathrm{mrad}$ or $19.6 \mathrm{mrad}$ were used as indicated. Care was taken to ensure the same probe current was used for each convergence angle by adjusting the monochromator gun lens bias. For beam current measurements, a Gatan UltraScan XP CCD was calibrated using a picoammeter connected to the EELS drift tube[16, 17]. As measured using the calibrated CCD, a 30 pA beam current was maintained to ensure minimal sample damage during mapping. Further, HAADF images were acquired before and after spectral mapping acquisition to monitor beam damage. Position averaged convergent beam electron diffraction (PACBED) was used for thickness determination at each EDX acquisition area [18].

X-ray maps were acquired using the FEI Super-X detector, with a 0.7 sr nominal collection solid angle, paired with the Bruker Espirit software. Each $68 \times 68$ pixel map was acquire for $600 \mathrm{~s}$ (clock time) with 
drift correction applied. For dwell time studies, the same area on the specimen, with a thickness of 25 $\mathrm{nm}$, was imaged while maintaining a constant orientation of the specimen to minimize detector-specimen geometry effects. Further, the $\alpha$ and $\beta$ tilt angles were below 10 degrees to minimize shadowing by the sample holder[19, 20].

\subsection{Data processing}

Each element map was constructed by integrating the peak intensities of the Sr-K, Ti-K and O-K full shell signals, $\mathrm{K}_{\alpha+\beta}$. The raw map signals were extracted and averaged into a single unit cell using an initial map size of $68 \times 68$ pixels consisting of $4 \times 4$ unit cells [14] in order to increase the signal-to-noise ratio in each atomic resolution EDX map. Other than template averaging, no other processing was applied to the experiment data. Although avoiding lattice averaging is preferable to preserve elemental fluctuations in individual atom columns, in this study, lattice averaging provides a method for observing the effects of experimental parameters with higher precision. The $\mathrm{AC}$ and non-AC signals were determined by fitting 2-D Gaussian distributions [21]. For dwell time measurements, the reported error, $\sigma$, was determined using Poisson statistics.

\subsection{Simulations}

The $\mu \mathrm{STEM}$ program was used to simulate X-ray maps using the measured microscope and specimen parameters [22]. The finite effective source size was taken into account by applying a 1.6 A FWHM Gaussian distribution, which gave the best match to the experimental data [23, 24]. Although aberration-corrected STEM probes routinely achieve sub-Ångström resolution, the large $1.6 \AA$ effective source size approximately accounts for the drift along the $z$ axis during EDX acquisition and misalignment between averaged spectral frames.

Simulations were used to estimate the portion of the amorphous surface non-AC signal, which was then added to the crystal simulations. As a first approximation to include amorphous surface layers, additional simulations were run using $\mathrm{SrTiO}_{3}$ with each atom randomly displaced in the structure [25]. This approximation was used to keep the computing power and time manageable. The approach was verified by simulating a few test-case supercells with amorphous surface layers capping the crystalline bulk. Both methods were in agreement between the $\mathrm{AC}$, non- $\mathrm{AC}$ and mean intensity signals. Using this approach, the best fit between experiment and simulation occurred when a $2 \mathrm{~nm}$ amorphous layer was present on each specimen surface. 

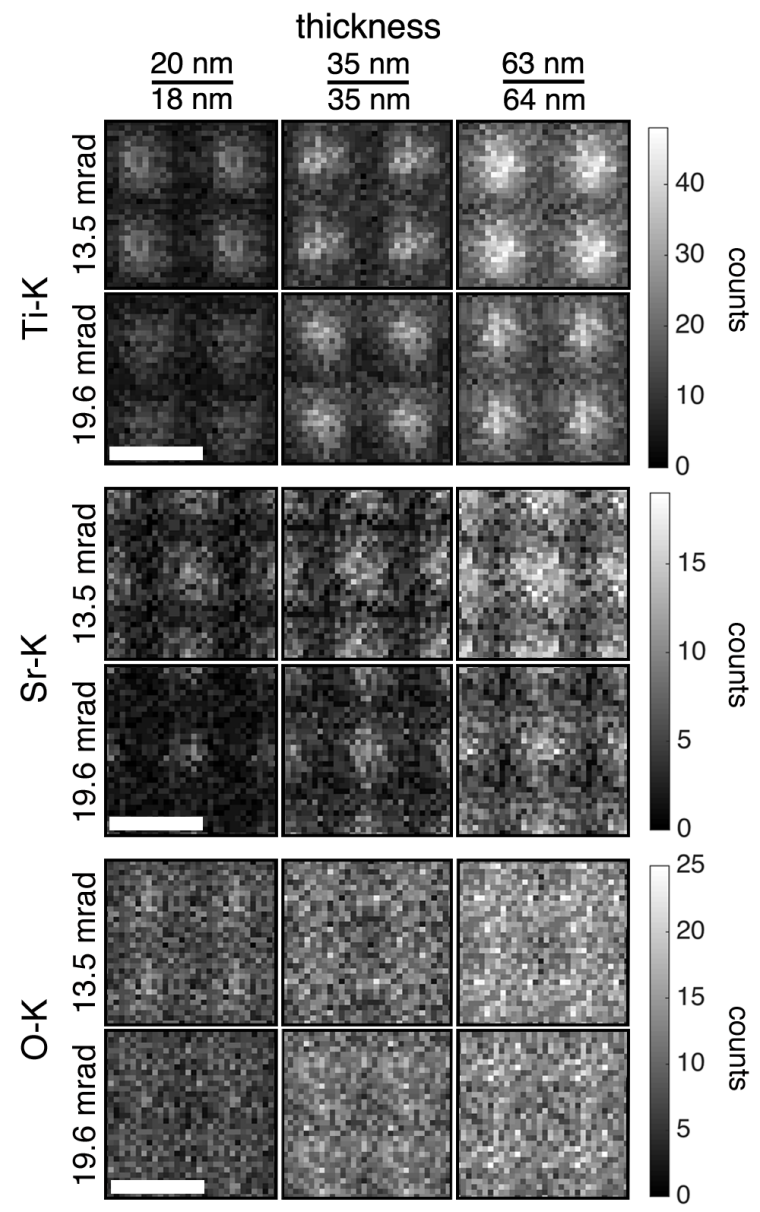

Figure 1: Atomically resolved EDX signal from experiment for Ti-K, Sr-K, and O-K as a function of convergence angle and thickness. Each map has been lattice averaged with a single unit cell template. The scale bar indicates 3.9 Å, the lattice parameter of STO. 


\section{Results and discussion}

\subsection{Convergence angles and thickness}

As an initial overview of the influence of convergence angle, atomically resolved EDX maps as a function of thickness and two different convergence angles are shown in Fig. 1. For direct comparison, the same probe current was used throughout, thereby ensuring that the X-ray counts for different convergence apertures are directly comparable. A cursory overview reveals that thicker crystal areas produce a higher total number of Ti and Sr X-ray counts, reaching a higher level for the $13.5 \mathrm{mrad}$ convergence angle than the $19.6 \mathrm{mrad}$. Close investigation reveals that the non-AC signal begins to degrade the AC-to-non-AC ratio with increasing thickness. As a result, the column-column resolvability is effectively reduced. This degraded atomic EDX signal is caused by spreading of the electron probe caused by increasing geometric spreading and multiple scattering with increasing thickness. Therefore, a trade off exists between obtaining sufficient counts to provide an acceptable signal-to-noise ratio and limiting the non-AC signal to maintain image contrast.

To understand the influence of experimental parameters on maintaining atom column contrast while obtaining sufficient X-ray signal, the AC and non-AC signals from each map have been extracted from the dataset using a 2D Gaussian fit for both experimental and simulated data, as schematically shown in Fig. 2(a). The AC and non-AC counts are then extracted from the fits, which correspond to the column regions shown in the blue box in Fig. 2(a). The non-AC represents the counts between atom columns. The column intensity represents the number of counts above the non-AC level. The AC, mean and non-AC counts for each convergence angle and thickness are shown for the Ti-K and Sr-K signals in Fig. 2(b,c). Each signal is normalized relative to the maximum ac intensity for both experimental and simulated results. Though this work is not reported on an absolute intensity scale, which is shown by Chen et. al.[26], the relative $\mathrm{AC}$ and non- $\mathrm{AC}$ intensities provide the influence from experimental parameters which allow for obtaining optimal conditions. For both Ti-K and Sr-K, each signal type is higher for a 13.5 mrad probe at each thickness. The non-AC counts show a nearly linear increase with thickness. The AC counts on the other hand experience a growth resembling a logarithmic relationship with a rapid increase with increasing thickness for thin conditions, but the growth decays with increasing thickness.

The mean count intensity trend reflects the contribution from both $\mathrm{AC}$ and non-AC signals. For thin samples, the signal for both convergence angles is similar. For thick specimens, however, the difference in channeling and geometric beam spreading conditions becomes pronounced for the two convergence angles. At thicker conditions, a larger fraction of the measured X-ray intensity resides in the non-AC. We further observe a difference in $\mathrm{AC}$ to non- $\mathrm{AC}$ behavior for different elements. For heavier elements, Sr in this case, we observe a greater fraction of the X-ray signal in the non- $\mathrm{AC}$ signal at thick regions than the non-AC from Ti-K.

As we observe differences in the behavior of $\mathrm{AC}$ and non- $\mathrm{AC}$ signals for convergence angle, thickness, 


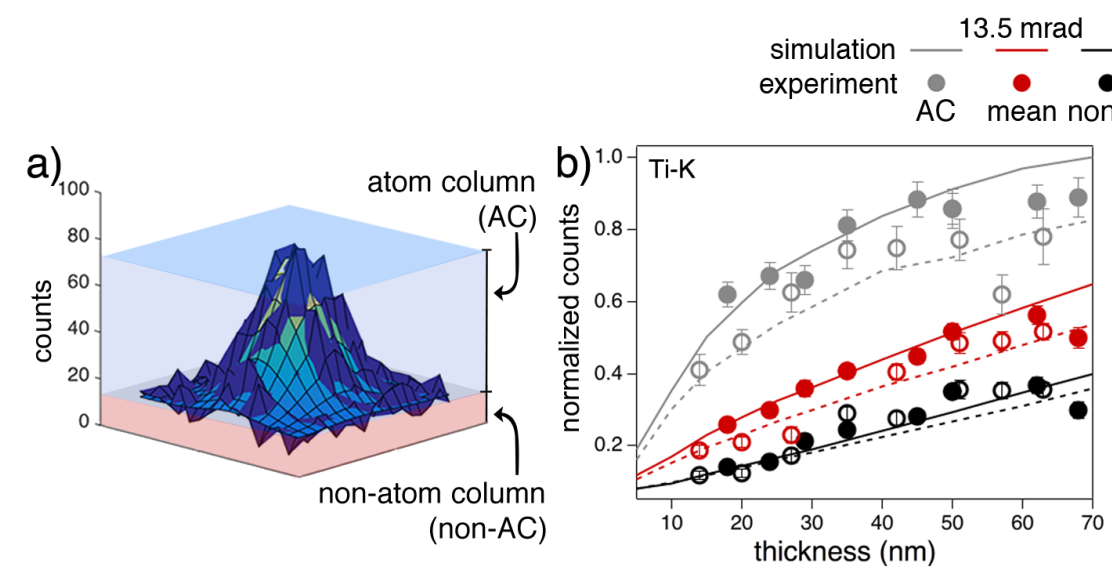

19.6 mrad

000

AC mean non-AC AC mean non-AC
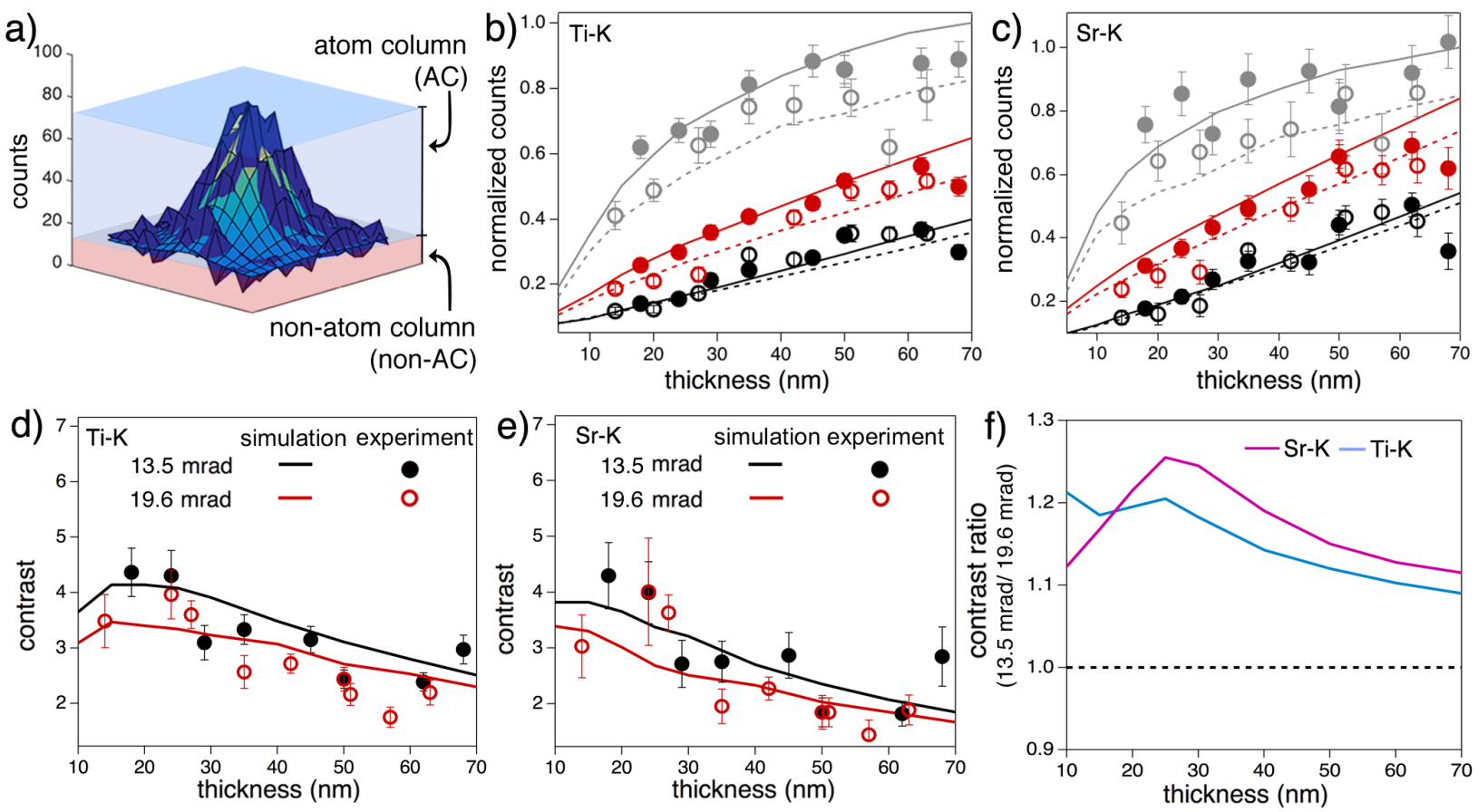

Figure 2: Atomic resolution EDX signals collected from experiment and simulation. (a) Example of the experimental lattice averaged Ti signal acquired using a $13.5 \mathrm{mrad}$ convergence semi-angle with an overlaid 2-D Gaussian fit. The red and blue planes indicate the $\mathrm{AC}$ and non-AC signals. (b-c) Experimental and simulated AC, mean, and non-AC signals from atomic resolution EDX maps for Ti-K (b) and Sr-K (c) for convergence semi-angles of $13.5 \mathrm{mrad}$ and $19.6 \mathrm{mrad}$. (d-e) Contrast ratios for Ti-K (d) and Sr-K (e). (f) Ratio of the contrast for $13.5 \mathrm{mrad}$ divided by the contrast for $19.6 \mathrm{mrad}$. Both plots are consistently above the dashed line reference at unity, indicating that the $13.5 \mathrm{mrad}$ probe provides consistently greater contrast than the $19.6 \mathrm{mrad}$ probe. 
and atom column composition, we quantify the atom column to non-AC, henceforth referred to as contrast. The contrast, $C$, can simply be defined:

$$
C=\frac{I_{A C}}{I_{n o n-A C}},
$$

with $I_{A C}$ being the intensity from the AC above the non-AC intensity, $I_{n o n-A C}[25]$.

The contrast is shown as a function of thickness for both simulation and experiment in Fig. 2(d-e). The contrast changes can be attributed to a difference in channeling conditions. For lower convergence angles, the channeling length is longer than for high convergence angles. Thus, the electron beam will travel along an atom column longer for lower convergence angles, increasing the contribution to the $\mathrm{AC}$ signal. Once dechanneling occurs, the beam spreads into the background, increasing the non-AC signal. In both cases, the contrast decreases as thickness increases because of the increased non-AC signal from beam spreading. The contrast ratio for $13.5 \mathrm{mrad}$ relative to $19.6 \mathrm{mrad}$ yields a clearer visualization of the behavior, as shown in Fig. 2(f). For all thicknesses, the smaller convergence angle is shown to provide a higher contrast. Moreover, the improvement is shown to be at least $10 \%$ for all thicknesses and up to $27 \%$ higher for the Sr-K signal at $30 \mathrm{~nm}$. The contrast is also shown to change for different atom columns. For example, the maximum and minimum contrast in the range shown is 4.2 and 2.3 for Ti-K and 3.8 and 1.9 for Sr-K. Although increased channeling serves to increase contrast, it is important to note that channeling affects the analysis of elemental composition and is essential to consider when performing quantitative atomically resolved EDX, as shown by Chen et. al. [26].

Considering the strong agreement between experimental and simulated data, simulated EDX maps for a range of convergence angles and thicknesses have been performed to find optimal operating conditions for $\mathrm{SrTiO}_{3}$ at $200 \mathrm{keV}$. A series of convergence angles between $5 \mathrm{mrad}$ and $30 \mathrm{mrad}$ with a thickness range of 10 $\mathrm{nm}-70 \mathrm{~nm}$ was simulated. Example atomic EDX maps of the thickness-convergence angle dependence are shown in Fig. 3.

While the total intensity, including both $\mathrm{AC}$ and non-AC signals, simply increases with increasing thickness for the Ti-K and Sr-K maps in Fig. 3, the convergence angle dependence is not so simple. With a convergence angle of $8 \mathrm{mrad}$, the non-AC intensity is much higher than any of the other convergence angles, resulting in a contrast that is much poorer than at larger convergence angles. At $8 \mathrm{mrad}$ in the case shown, the probe becomes broad to the extent that significant overlap occurs between AC signals, resulting in very high non-AC counts. For the maps shown, the signals reach a maximum column intensity at approximately $12 \mathrm{mrad}$.

As the convergence angle is increased above $12 \mathrm{mrad}(16 \mathrm{mrad}$ and $19.6 \mathrm{mrad})$ in the maps shown in Fig. 3, the intensity decreases. The maximum column intensity at $12 \mathrm{mrad}$ results from the incorporation of the finite effective source size. Without incorporation of the source size, the maximum AC intensity occurs 
at a higher convergence angle $(19.6 \mathrm{mrad})$. Since the column peaks at $19.6 \mathrm{mrad}$ are narrower than $12 \mathrm{mrad}$, however, the intensity at atom columns at $19.6 \mathrm{mrad}$ becomes lower than $12 \mathrm{mrad}$ upon incorporation of the finite source size.
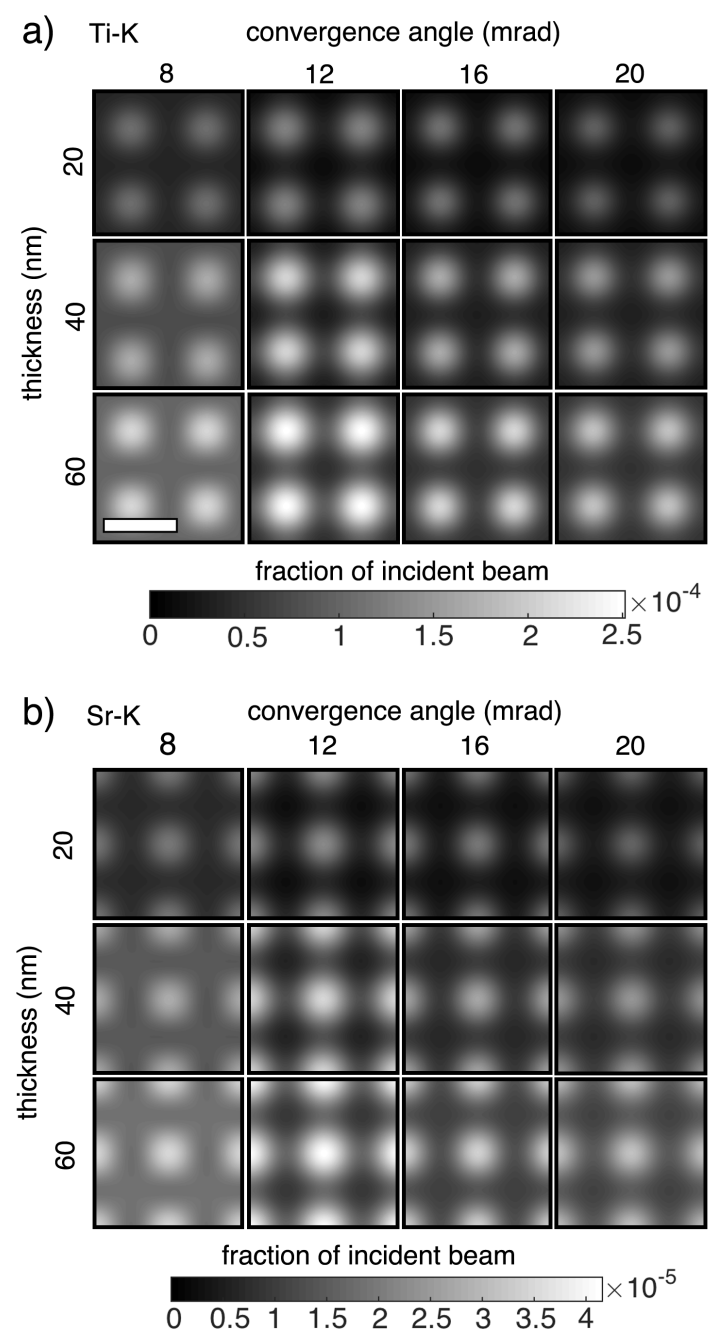

Figure 3: Simulated atomically resolved EDX maps for varying thickness and convergence angles for (a) Ti-K and (b) Sr-K. The scale bar represents $3.9 \AA$.

To quantitatively address the influence of the convergence angle and thickness, intensity and contrast maps have been generated. By plotting contrast for each thickness and convergence angle, Fig. 4 (a) Ti-K and (b) Sr-K, we observe the optimal operating conditions for atomic column contrast for $\mathrm{SrTiO}_{3}$ at 200 $\mathrm{keV}$. To achieve ideal operating conditions, we must maintain strong contrast of the atom columns in EDX maps for visibility of elemental signals and also collect sufficient counts. The X-ray intensity is shown in Fig. 4 for (c) Ti-K and (d) Sr-K signals. As the convergence angle increases, the AC and non-AC intensity is 
observed to decrease.

The trend in contrast is observed to be different from that of the total X-ray signals, as expected from the example maps shown in Fig. 3. For the Ti-K and Sr-K signals, the contrast will increase with increasing convergence angle until the probe is narrower than the effective scattering potential at extremely thin conditions (under $5 \mathrm{~nm}$ ). As the thickness increases beyond $10 \mathrm{~nm}$, which is generally needed to improve the signal-to-noise ratio, the optimal convergence angle that maximizes contrast moves to smaller convergence angles. The contrast also exhibits a maximum for certain convergence angles. This occurs because at very low convergence angles significant overlap in signal from atom columns occurs, causing highly increased non-AC signal. At the other end of the range, i.e. large convergence angles, the electron probe is less localized to atom columns, resulting in a higher percentage of X-rays being generated within the non-local background. An optimal convergence angle range therefore occurs once the spatial resolution can sufficiently resolve each atom column. For the case of $\mathrm{SrTiO}_{3}$ at $200 \mathrm{keV}$ and the effective source size of the microscope, the optimal convergence angle for samples with thickness of $10 \mathrm{~nm}$ and above is approximately $13 \mathrm{mrad}$.
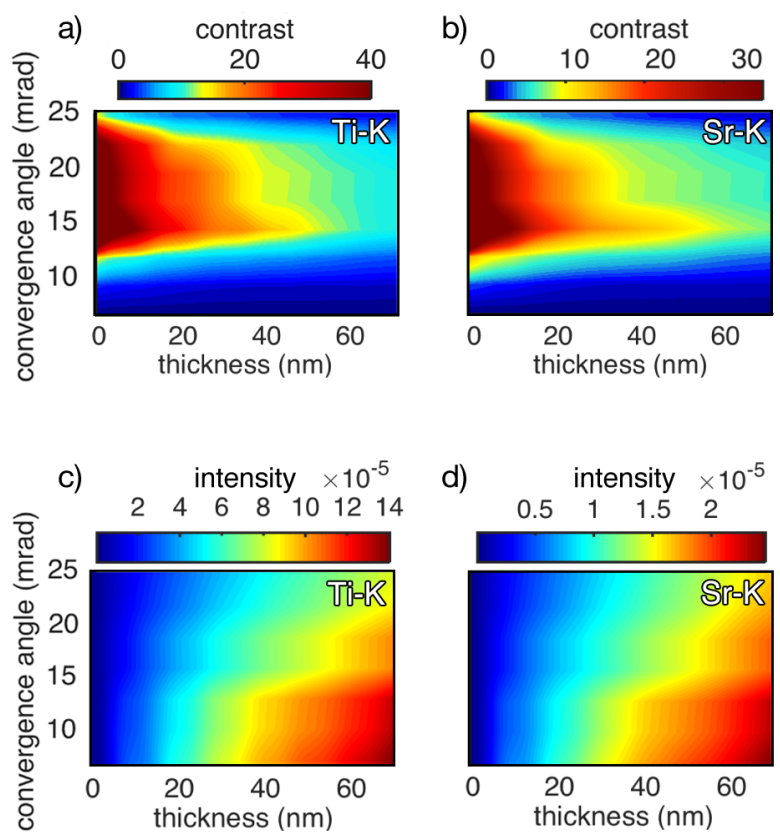

Figure 4: Atomically resolved EDX atom column contrast (a-b) and the number of relevant ionization events caused as a fraction of the incident beam (c-d) as a function of convergence angle and thickness for Ti and Sr.

While an optimal convergence angle was determined for $\mathrm{SrTiO}_{3}$, this parameter is expected to vary depending on the crystal structure. For example, samples with more closely packed atom columns should yield an optimal convergence angle greater than $13 \mathrm{mrad}$ in order to sufficiently resolve the atom columns. To serve as a test system, $\gamma^{\prime}-\mathrm{Ni}_{3} \mathrm{Al}<001>$ is chosen due to the tighter spacing between nearest neighbor Ni-Ni 
atom columns $\left(1.78 \AA\right.$ ), Figure 5 (a), than nearest neighbor Sr-Sr or Ti-Ti columns in $\mathrm{SrTiO}_{3}(3.905 \AA$ ). Simulations to determine the X-ray signal and atom column contrast for a series of convergence angles and thickness, similar to Figure 4 for $\mathrm{SrTiO}_{3}<001>$, are shown in Figure 5. For the Ni contrast, Figure 5(b), the optimum simulated convergence angle is $18 \mathrm{mrad}$ for thicknesses between $20 \mathrm{~nm}$ and $45 \mathrm{~nm}$. Alternatively, the optimal convergence angle for increased contrast in the $\mathrm{Al}$ signal, Figure 5 (c), is 13 mrad for thicknesses above $20 \mathrm{~nm}$. The AC and non-AC intensity again increases for an increasing thickness and decreasing convergence angle, Figure 5 (d-e). For the Al signal with a relatively large lattice spacing compared to Ni, the optimum convergence angle is similar to the case of $\mathrm{SrTiO}_{3}$. The tight spacing of Ni columns, however, requires a higher convergence angle to reduce overlap between AC signals.

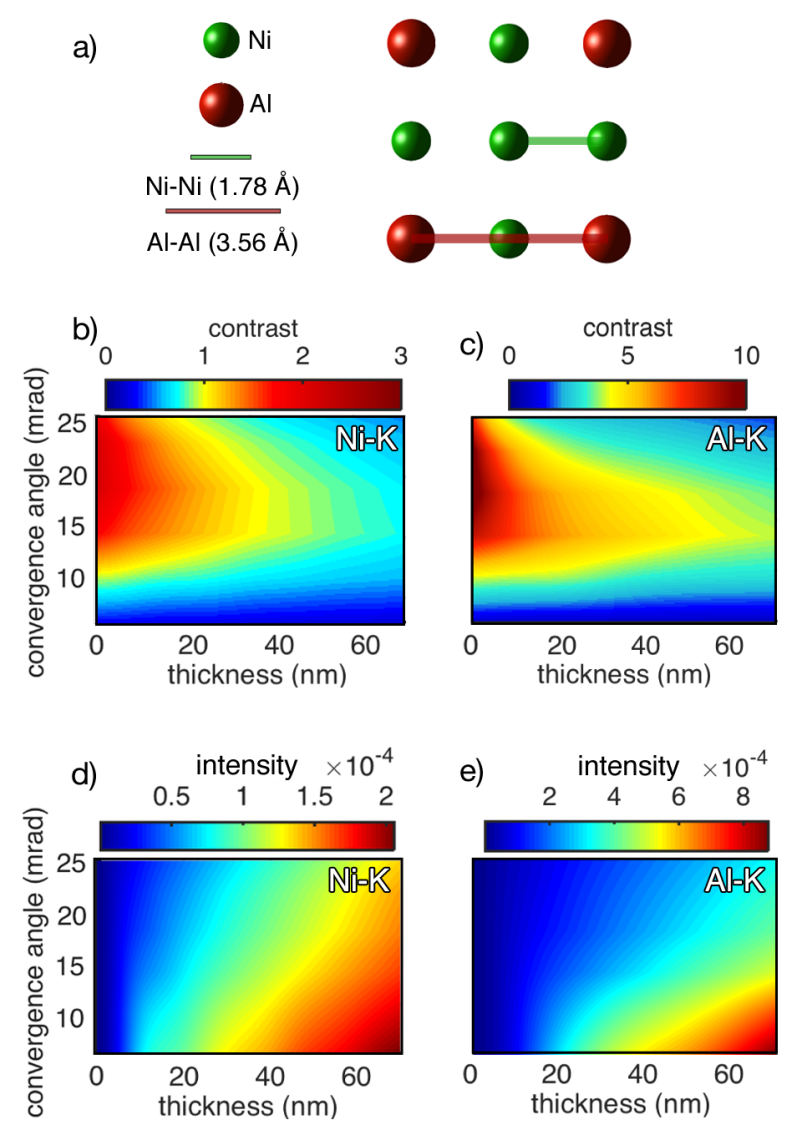

Figure 5: Schematic of the ordered $\gamma^{\prime}-\mathrm{Ni}_{3} \mathrm{Al}<001>$ structure with indicated Ni-Ni (green) and Al-Al (red) atom column separations (a). Atom column contrast (b-c) and X-ray yield as a fraction of the electron beam (d-e) as a function of convergence angle and thickness for $\mathrm{Ti}$ and $\mathrm{Sr}$.

\subsection{Dwell time}

Having optimized experimental parameters that affect the generation of X-rays, it is important to ensure as many of the X-rays are collected as possible. For the Super-X four quadrant detector, it is well known 
a)
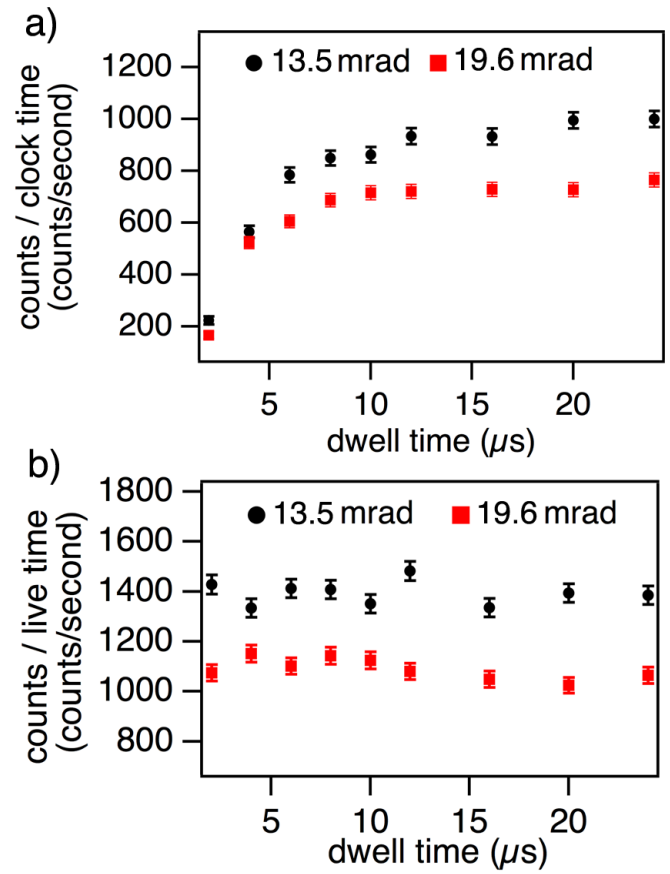

c)

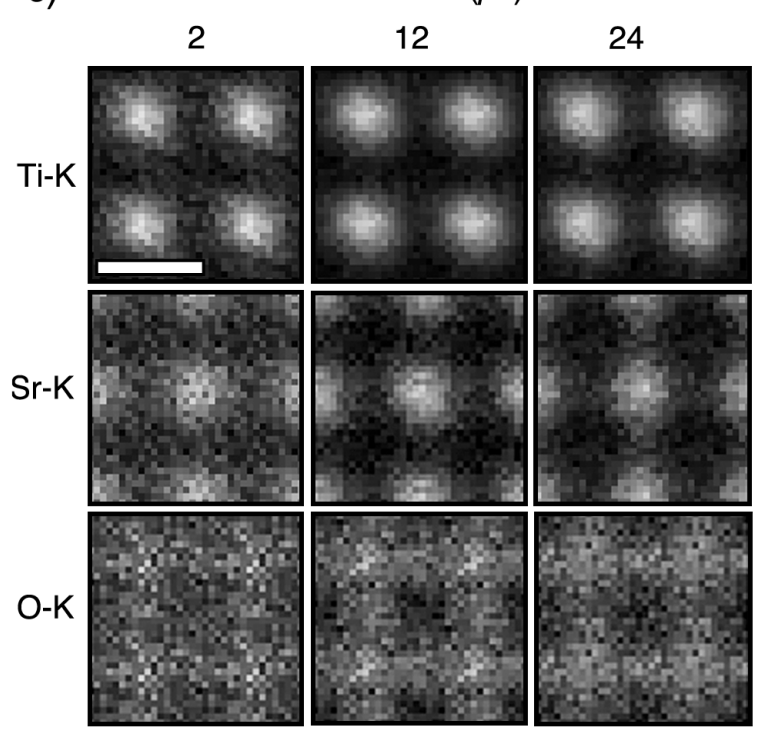

Figure 6: Effects of dwell time in atomically resolved EDX. (a) Total processed X-ray counts collected per second of clock time in an EDX map for 13.5 mrad and 19.6 mrad convergence angles plotted as a function of dwell time. Counts are integrated from the entire 2-D map and energy range. (b) Counts processed per second of live time.

(c) X-ray elemental maps collected with varying the dwell time using a convergence angle of 13.5 mrad with intensities based on the counts in each individual map. Each map was taken at a sample thickness of $25 \mathrm{~nm}$.

The scale bar represents $3.9 \AA$. 
that sample tilt should be minimal in order to limit shadowing effects from the sample holder $[12,19,20,27]$. An additional parameter for increasing the total signal in EDX mapping which can easily be controlled is increasing the time that the beam spends at each pixel. Although the total generated X-ray count rate will be the same for a given thickness, software acquisition parameters will affect the total processed X-ray signal. It is important to note that detector time constant for the Super-X detector is set to a fixed value by the manufacturer [28].

By changing the probe dwell time, the time that the beam is positioned on a pixel per frame, the processed $\mathrm{X}$-ray count rate is measured as shown in Figure 6(a). Initially at low dwell times, $2 \mu \mathrm{s}$, the processed EDX count rate is $\sim 200$ counts per second. Once the dwell time is increased to $\sim 10 \mu \mathrm{s}$, the count rate is increased by roughly a factor of four. The processed counts become approximately constant after this initial gain, with fewer counts gained from an increased dwell time. The time constant for the microscope is fixed at $16 \mu \mathrm{s}$. Note that the time constant, or shaping time, is merely the time that the system processes the incoming $\mathrm{X}$-ray, not the time that the system waits for an incoming X-ray. For example, a time constant of $16 \mu \mathrm{s}$ and count rate of 1000 counts/second results in a dead time of $1.6 \%$. The increase in counts at higher dwell times are thus due to other reasons, which can be explained through several factors.

The probe transit time between pixels remains the same regardless of dwell time, which results in an excessive time without X-ray collection for short dwell times. In addition, the beam fly back will increase time without X-ray collection [29]. For short dwell times, more frames will be collected within the same period of time, resulting in many more occurrences of beam fly back and drift correction. To ensure that the loss in signal occurs due to increased inactivity of X-ray processing, the counts are plotted as a function of the live time, or the actual time that X-rays have been processed, in Figure 6(b). The count rate for the live time is shown to be constant for any dwell time, which has important experimental implications. For example, to obtain the same number of total counts at $2 \mu s$ as at $20 \mu s$, the clock time would have to be increased by nearly a factor of 10 . As a result, the sample may drift out of range or focus. Furthermore, it is critical that the same live time be used, even though dead time is near zero, when comparing total counts between maps.

The effects of the increased processed X-ray count rate can be observed in Figure 6(b). Elemental maps for Ti-K, Sr-K, and O-K are reported as a function of the dwell time for each pixel in the atomic resolution EDX maps shown in Figure 6(b). The signal-to-noise varies for each element due to factors such as the ionization cross section, fluorescence yields, and detector efficiencies at different energies [30,31,32]. At short dwell times, $2 \mu$ s in this case, the noise level is much higher than for longer dwell times because the clock time is the same. By increasing the dwell time from $12 \mu \mathrm{s}$ to $24 \mu$ s however, the noise reduction is marginal and $24 \mu$ s maps may in fact be more blurred than $12 \mu$ s maps. This could be due to a longer total time between drift correction for the $24 \mu \mathrm{s}$ maps, causing difficulties in the drift correction between spectral frames. From the results, one should use a dwell time above $10 \mu$ s or otherwise forfeit a large number of 
counts in the spectra for a fixed clock time. The loss of processed counts at short dwell times is an important consideration for fast EDX mapping, where frames are acquired very quickly to reduce beam damage and drift. Although beam damage can be decreased at shorter dwell times, processed counts will be lost as well. On the other hand, long dwell times will result in less confidence in the cross correlation between frames. For example, with a sufficiently large drift rate the sample can move more than half a unit cell for one frame, the cross correlation may not restore the correct atom column to the initial position, causing misrepresentation in elemental fluctuation.

\section{Conclusions}

Beyond improvements in collection solid angle enabled by state-of-the-art EDX detectors, the key experimental parameters investigated, convergence angle, thickness and dwell time, have been shown to play a critical role for providing high quality atomic resolution EDX mapping. We have shown that optimizing the probe forming convergence semi-angle can increase the obtainable signal, increasing the signal-to-noise ratio as well contrast. In reciprocal space, this optimal condition is equivalent to causing sufficient, but not excessive, diffraction disc overlap. Compounding the increased contrast, up to $27 \%$, and improving signal-to-noise due to the $20-30 \%$ increase in total counts using a smaller convergence angle, greatly improves both detectability and quantitative statistical analysis for atomic resolution EDX maps. Convergence angles that are too small, however, cause signal overlap between atom columns, degrading contrast. Importantly, the optimal convergence semi-angle is structure dependent. For structures with shorter column-to-column distances, a larger convergence angle is needed to reduce signal overlap between atom columns. Finally, the selected dwell time also plays a critical role for collecting sufficient signal for resolving atomic resolution elemental signal in EDX mapping. Overall, these results can provide important guidelines for future work in identifying the elemental makeup of atom columns in EDX mapping.

\section{Acknowledgments}

JHD, WZ, XS, and JML gratefully acknowledge support from the Air Force Office of Scientific Research (Grant No. FA9550-14-1-0182). JHD acknowledges support for this work by the National Science Foundation Graduate Research Fellowship (Grant DGE-1252376). This project was supported under the Discovery Projects funding scheme of the Australian Research Council (Projects DP140102538 and DP110102228). AJD acknowledges the Discovery Early Career Researcher Award from the Australian Research Council (Project No. DE130100739). The authors acknowledge the use of the Analytical Instrumentation Facility (AIF) at North Carolina State University, which is supported by the State of North Carolina and the National Science Foundation. 


\section{References}

[1] A. J. D'Alfonso, B. Freitag, D. Klenov, L. J. Allen, Atomic-resolution chemical mapping using energy-dispersive x-ray spectroscopy, Phys. Rev. B 81 (2010) 100101.

[2] M.-W. Chu, S. C. Liou, C.-P. Chang, F.-S. Choa, C. H. Chen, Emergent chemical mapping at atomic-column resolution by energy-dispersive X-ray spectroscopy in an aberration-corrected electron microscope, Phys. Rev. Lett. 104 (2010) 196101.

[3] D. O. Klenov, J. M. Zide, Structure of the InAlAs/InP interface by atomically resolved energy dispersive spectroscopy, Appl. Phys. Lett. 99 (2011) 141904.

[4] J. H. Dycus, R. M. White, J. M. Pierce, R. Venkatasubramanian, J. M. LeBeau, Atomic scale structure and chemistry of $\mathrm{Bi}_{2} \mathrm{Te}_{3} / \mathrm{GaAs}$ interfaces grown by metallorganic van der Waals epitaxy, Appl. Phys. Lett. 102 (2013) 081601.

[5] G. Kothleitner, M. J. Neish, N. R. Lugg, S. D. Findlay, W. Grogger, F. Hofer, L. J. Allen, Quantitative elemental mapping at atomic resolution using X-ray spectroscopy, Phys. Rev. Lett. 112 (2014) 085501.

[6] J. H. Dycus, J. S. Harris, X. Sang, C. M. Fancher, S. D. Findlay, A. A. Oni, T.-t. E. Chan, C. C. Koch, J. L. Jones, L. J. Allen, D. L. Irving, J. M. LeBeau, Accurate nanoscale crystallography in real-space using scanning transmission electron microscopy, Microscopy and Microanalysis 21 (2015) 946-952.

[7] P. Lu, L. Zhou, M. J. Kramer, D. J. Smith, Atomic-scale chemical imaging and quantification of metallic alloy structures by energy-dispersive x-ray spectroscopy, Sci. Rep. 4 (2014) 3945.

[8] P. Lu, J. Xiong, M. Van Benthem, Q. Jia, Atomic-scale chemical quantification of oxide interfaces using energy-dispersive X-ray spectroscopy, Appl. Phys. Lett. 102 (2013) 173111.

[9] P. G. Kotula, D. O. Klenov, H. S. von Harrach, Challenges to quantitative multivariate statistical analysis of atomicresolution X-ray spectral, Microscopy and Microanalysis 18 (2012) 691-698.

[10] Z. Chen, A. J. D'Alfonso, M. Weyland, D. J. Taplin, L. J. Allen, S. D. Findlay, Energy dispersive x-ray analysis on an absolute scale in scanning transmission electron microscopy, Ultramicroscopy 157 (2015) 21-26.

[11] M. Watanabe, D. B. Williams, The quantitative analysis of thin specimens: a review of progress from the Cliff-Lorimer to the new $\zeta$-factor methods, Journal of Microscopy 221 (2) (2006) 89-109.

[12] C. S. M. Yeoh, D. Rossouw, Z. Saghi, P. Burdet, R. K. Leary, P. A. Midgley, The dark side of EDX tomography: Modeling detector shadowing to aid 3D elemental signal analysis, Microscopy and Microanalysis 21 (2015) 759-764.

[13] B. D. Forbes, A. J. D’Alfonso, R. E. A. Williams, R. Srinivasan, H. L. Fraser, D. W. McComb, B. Freitag, D. O. Klenov, L. J. Allen, Contribution of thermally scattered electrons to atomic resolution elemental maps, Phys. Rev. B 86 (2) (2012) 024108.

[14] P. Lu, E. Romero, S. Lee, J. L. MacManus-Driscoll, Q. Jia, Chemical quantification of atomic-scale EDS maps under thin specimen conditions, Microscopy and Microanalysis 20 (2014) 1782-1790.

[15] P. M. Voyles, J. L. Grazul, D. A. Muller, Imaging individual atoms inside crystals with ADF-STEM, Ultramicroscopy $96(3-4)(2003) 251-273$

[16] R. Ishikawa, A. R. Lupini, S. D. Findlay, S. J. Pennycook, Quantitative annular dark field electron microscopy using single electron signals, Microscopy and Microanalysis 20 (2014) 99-110.

[17] X. Sang, J. LeBeau, Characterizing the response of a scintillator-based detector to single electrons, Ultramicroscopy 161 (2016) 3-9.

[18] J. M. LeBeau, S. D. Findlay, L. J. Allen, S. Stemmer, Position averaged convergent beam electron diffraction: Theory and applications, Ultramicroscopy 110 (2) (2010) 118-125.

[19] T. J. Slater, A. Janssen, P. H. Camargo, M. G. Burke, N. J. Zaluzec, S. J. Haigh, STEM-EDX tomography of bimetallic nanoparticles: A methodological investigation, Ultramicroscopy 162 (2016) 61-73.

[20] W. Xu, J. Dycus, X. Sang, J. LeBeau, A numerical model for multiple detector energy dispersive x-ray spectroscopy in the transmission electron microscope, Ultramicroscopy 164 (2016) 51-61. 
[21] X. Sang, A. A. Oni, J. M. LeBeau, Atom column indexing: Atomic resolution image analysis through a matrix representation, Microscopy and Microanalysis 20 (2014) 1764-1771.

[22] L. J. Allen, A. J. D'Alfonso, S. D. Findlay, Modelling the inelastic scattering of fast electrons, Ultramicroscopy 151 (0) (2015) $11-22$

[23] J. M. LeBeau, S. D. Findlay, L. J. Allen, S. Stemmer, Quantitative atomic resolution scanning transmission electron microscopy, Phys. Rev. Lett. 100 (20) (2008) 206101.

[24] C. Dwyer, C. Maunders, C. L. Zheng, M. Weyland, P. C. Tiemeijer, J. Etheridge, Sub-0.1nm-resolution quantitative scanning transmission electron microscopy without adjustable parameters, Appl. Phys. Lett. 100 (19) (2012) 191915.

[25] K. Mkhoyan, S. Maccagnano-Zacher, E. Kirkland, J. Silcox, Effects of amorphous layers on ADF-STEM imaging, Ultramicroscopy 108 (8) (2008) $791-803$.

[26] Z. Chen, M. Weyland, X. Sang, W. Xu, J. Dycus, J. LeBeau, A. D'Alfonso, L. Allen, S. Findlay, Quantitative atomic resolution elemental mapping via absolute-scale energy dispersive x-ray spectroscopy, Ultramicroscopy 168 (2016) 7 - 16.

[27] P. Schlossmacher, D. O. Klenov, B. Freitag, S. von Harrach, A. Steinbach, Nanoscale chemical compositional analysis with an innovative S/TEM-EDX system, Microscopy and analysis (142) (2010) S5.

[28] J. Rignalda, private communication (April 27 2016).

[29] P. Miller, private communication (April 28 2016).

[30] L. J. Allen, A. J. D’Alfonso, B. Freitag, D. O. Klenov, Chemical mapping at atomic resolution using energy-dispersive X-ray spectroscopy, MRS Bulletin 37 (2012) 47-52.

[31] A. Kahoul, V. Aylikci, N. K. Aylikci, E. Cengiz, G. Apaydin, Updated database and new empirical values for K-shell fluorescence yields, Radiation Physics and Chemistry 81 (2012) $713-727$.

[32] A. Thompson, D. Attwood, E. Gullikson, M. Howells, J. Kortright, A. Robinson, J. Underwood, K. Kim, J. Kirz, I. Lindau, P. Pianetta, H. Winick, G. Williams, J. Scofield, X-ray data booklet, Lawrence Berkeley National Laboratory, University of California Berkeley, California 94720 (January 2001). 